\title{
Neurochemical metabolites in prefrontal cortex in patients with mild/moderate levels in first-episode depression
}

This article was published in the following Dove Press journal:

Neuropsychiatric Disease and Treatment

9 August 2013

Number of times this article has been viewed

\author{
Gülfizar Sözeri-Varma' \\ Nalan Kalkan-Oğuzhanoglu' \\ Muharrem Efe' \\ Yılmaz Kıroglu ${ }^{2}$ \\ Taçlan Duman' \\ 'Department of Psychiatry, \\ ${ }^{2}$ Department of Radiology, Faculty \\ of Medicine, Pamukkale University, \\ Denizli, Turkey
}

Background: Previous studies have determined the neurochemical metabolite abnormalities in major depressive disorder (MDD). The results of studies are inconsistent. Severity of depression may relate to neurochemical metabolic changes. The aim of this study is to investigate neurochemical metabolite levels in the prefrontal cortex (PFC) of patients with mild/moderate MDD.

Methods: Twenty-one patients with mild MDD, 18 patients with moderate MDD, and 16 matched control subjects participated in the study. Patients had had their first episode. They had not taken treatment. The severity of depression was assessed by the Hamilton Rating Scale for Depression (HAM-D). Levels of $N$-acetyl aspartate (NAA), choline-containing compounds (Cho), and creatine-containing compounds $(\mathrm{Cr})$ were measured using proton magnetic resonance spectroscopy ( $\left.{ }^{1} \mathrm{H}-\mathrm{MRS}\right)$ at $1.5 \mathrm{~T}$, with an $8-\mathrm{cm}^{3}$ single voxel placed in the right PFC.

Results: The moderate MDD patients had lower NAA/Cr levels than the control group. No differences were found in neurochemical metabolite levels between the mild MDD and control groups. No correlation was found between the patients' neurochemical metabolite levels and HAM-D scores.

Conclusion: Our findings suggest that NAA/Cr levels are low in moderate-level MDD in the PFC. Neurochemical metabolite levels did not change in mild depressive disorder. Our results suggest that the severity of depression may affect neuronal function and viability. Studies are needed to confirm this finding, including studies on severely depressive patients.

Keywords: major depressive disorder, magnetic resonance spectroscopy, $N$-acetyl aspartate, creatine, choline

\section{Introduction}

Major depressive disorder (MDD) is a syndrome that negatively affects a person's life and functionality. ${ }^{1}$ Functional neuroanatomical and structural magnetic resonance imaging (MRI) studies have shown abnormalities related to emotional processes in brain structures in MDD. Volume changes in the prefrontal cortex (PFC), hippocampus, and striatum have been reported. ${ }^{2-5}$ Damage to neurons and glia and decreases in their numbers and sizes have been shown in a postmortem study. ${ }^{6}$ Lorenzetti et al $^{7}$ have examined the structural MRI studies carried out during 2000 and 2007. It has been determined that in MDD, volume decrease in hippocampus, basal ganglia, orbitofrontal cortex, and $\mathrm{PFC}$ is a consistent result. It has especially been observed that regional brain volume has been significantly affected in studies evaluating patients with long sickness time and repeating episodes. In another systematic review and meta-analysis, publications prior to March 2011 have been examined. In this meta-analysis, it has been determined
Correspondence: Gülfizar Sözeri-Varma Pamukkale University Faculty of Medicine, Department of Psychiatry, Doktorlar Cd, No 42, 20100 Denizli, Turkey

Tel +902584440728 ext I 106

Fax +90258 24I 0040

Email gvarma@pau.edu.tr 
that there is volume decrease in brain regions such as frontal cortex, orbitofrontal cortex, cingulated cortex, hippocampus, and striatum in unipolar depressive disorder. ${ }^{8}$

Proton magnetic resonance spectroscopy $\left({ }^{1} \mathrm{H}-\mathrm{MRS}\right)$ is an imaging technique that can be used to measure neurochemical structures and the metabolites of tissues noninvasively and present them in one spectrum. This technology can be used to determine the in vivo levels of neurochemical compounds that contain $N$-acetyl aspartate (NAA), creatine $(\mathrm{Cr})$, and choline (Cho) in an evaluated region of interest. NAA is a compound that is found in adult neurons and is recognized as a marker of neuronal integrity, viability, and/or function. ${ }^{9}$ The resonance signal for Cho resonance is composed of phosphocholine, glycerophosphocholine, and free Cho. It has been argued that high Cho levels result from changes in the metabolism of phospholipids in cellular membranes and from a high degree of membrane degradation. Total $\mathrm{Cr}$, comprised of $\mathrm{Cr}$ and phosphocreatine, is a marker of oxidative metabolism. The creatine peak is used as a reference value because it remains consistent in H-MRS; this use of the $\mathrm{Cr}$ peak is based on the assumption that $\mathrm{Cr}$ resonance is constant and is not affected by various pathologies. ${ }^{10}$

The PFC plays an important role in mood regulation. ${ }^{11}$ The number of studies on neurochemical changes that occur in the PFC during depression is few and there are conflicting results. In patients with melancholic features and excessive depressive symptoms, metabolite levels before and after electroconvulsive treatment and pharmacotherapy have been examined. It has been determined that glutamate/glutamine levels were lower in comparison with the control group and that there is a negative correlation with depressive symptom severity. It has been emphasized that glutamate/glutamine have recovered following a successful treatment and that this anomaly is situational. ${ }^{12}$ The frontal cortex NAA/Cr, $\mathrm{Cho} / \mathrm{Cr}$, myo-inositol/Cr values in depression patients were determined to be lower in comparison with the healthy control group. However, the absolute $\mathrm{Cr}$ value in the patient group was determined to be higher in comparison with the control group. ${ }^{13}$ Coupland et al ${ }^{14}$ determined that levels of NAA, Cho, and $\mathrm{Cr}$ in the PFC were normal and myo-inositol levels were lower in patients with moderate or severe depressive disorder. They concluded that there are glial metabolism changes and glial cell loss in MDD. In a study carried out on elderly depression patients who have not been taking medication for at least 2 weeks, no statistically significant difference was determined in the NAA, Cho, Cr, glutamate/glutamine, and myo-inositol levels in comparison with the control group. ${ }^{15}$ In patients with chronic pain and depression, it was concluded that the lowness of NAA in the right dorsolateral PFC is related to depression and not to pain. ${ }^{16}$

In patients with unipolar depressive disorder, neurochemical metabolite levels such as dorsolateral PFC NAA, Cho, and $\mathrm{Cr}$ were determined to be similar to those of the healthy control group. However NAA/Cr levels were determined to be significantly lower in patients with longer sickness time in comparison to patients with shorter illness duration. ${ }^{16}$ It has also been reported that the levels of $\mathrm{Cr}$ and $\mathrm{Cho}$ in the hippocampus increase in patients with recurrent depressive disorder and this increase is related to both the number of depressive episodes and the duration of the disease. ${ }^{17}$ The severity of depression may be a factor that affects the changes in the neurochemical metabolite levels that are observed in MDD. ${ }^{18-20}$

The PFC is an important region for the regulation of emotion and conflicting results have been obtained in ${ }^{1} \mathrm{H}-\mathrm{MRS}$ studies carried out on this region. Previous studies lead one to think that depression severity and recurring depressive episodes affect neuronal functions and thus the study results. The objective of this study was to examine the neurochemical changes that occur on patients with only first-episode depression who do not use any medication. It is expected that the results obtained will contribute to the understanding of the neurochemical changes that occur in first-episode depressive disorder.

\section{Methods}

\section{Subjects and study procedure}

Subjects in this study were patients who were diagnosed with first-episode MDD according to diagnostic criteria from the Diagnostic and Statistical Manual of Mental Disorders, Fourth Edition (DSM-IV). ${ }^{21}$ This study was carried out on patients who had applied to the Pamukkale University Faculty of Medicine Psychiatry polyclinic from May 2010 to May 2011. A diagnostic assessment was performed to rule out other major Axis I disorders, including schizophrenia, bipolar disorders, and anxiety disorders. All of the patients were unmedicated. Subjects completed sociodemographic data forms that had been prepared by the researchers. Following psychiatric examination, the psychiatrist who was conducting the examination assessed the patients using the Hamilton Rating Scale for Depression (HAM-D, 17 items). The patients were informed about the study, and those who volunteered were included in the study. Patients with comorbid psychiatric disorders such as bipolar disorder, schizophrenia, or anxiety disorders were excluded from the study. Patients who were currently using psychotropic drugs, 
displayed psychotic symptoms and mental retardation, or had comorbidity of psychiatric or neurological diseases were excluded from the study. The control group was composed of healthy individuals from the hospital staff and their relatives whose age and gender were similar to the patient groups and who had no physical or mental disorders. For each patient, an appointment at the Pamukkale University Faculty of Medicine Radiology clinic was made, and ${ }^{1} \mathrm{H}-\mathrm{MRS}$ scans were completed within 1 week of psychiatric evaluation.

The patients with depression were divided into two groups based on their HAM-D scores. Subjects that received 15 or lower HAM-D scores were determined to have a mild level of MDD ( $\mathrm{n}=21)$, and those with HAM-D scores that were higher than 15 points were determined to have a moderate level of MDD $(n=18)$. In total, 39 patients with MDD and 16 healthy controls completed the study.

Approval for all procedures was obtained from the local ethics committee of Pamukkale University in accordance with the Declaration of Helsinki. Participants were informed about the study, and those who gave written consent were included.

\section{The Hamilton Rating Scale for Depression}

The HAM-D is a 17-item, clinician-administered scale that was first published in 1960. It can be used to assess the severity of a patient's depression; each item can be scored from 0 to $4 .^{22}$ Akdemir et $\mathrm{a}^{23}$ have tested the validity and reliability of the Turkish version of the HAM-D. Total scores that are between 0 and 7 indicate no depression; scores that are between 8 and 15 indicate mild depression; scores that are between 16 and 28 indicate moderate depression; and scores that are greater than 29 indicate severe depression. The highest possible score is 53 .

\section{Proton magnetic resonance spectroscopy}

The levels of various compounds in the right PFC were investigated using ${ }^{1} \mathrm{H}-\mathrm{MRS}$ (Figure 1). These compounds included NAA, Cr, and Cho, along with the NAA/Cr and Cho/Cr ratios. A ${ }^{1} \mathrm{H}-\mathrm{MRS}$ scan was conducted using a 1.5 Tesla magnetic resonance scanner (GE Medical Systems, Milwaukee, WI, USA) and a standard head coil. The magnetic resonance protocol was conducted in the coronal plane using a $\mathrm{T} 2$-weighted fast spin-echo sequence with a 10-mm thickness and the following parameters: time of repetition (TR)/time of echo (TE), 3000/85; field of view, 14; Matrix, $352 \times 352$; and Next, 1 . However, MRS was measured with a single voxel ( ${ }^{1} \mathrm{H}$-voxel). The ${ }^{1} \mathrm{H}-\mathrm{MRS}$

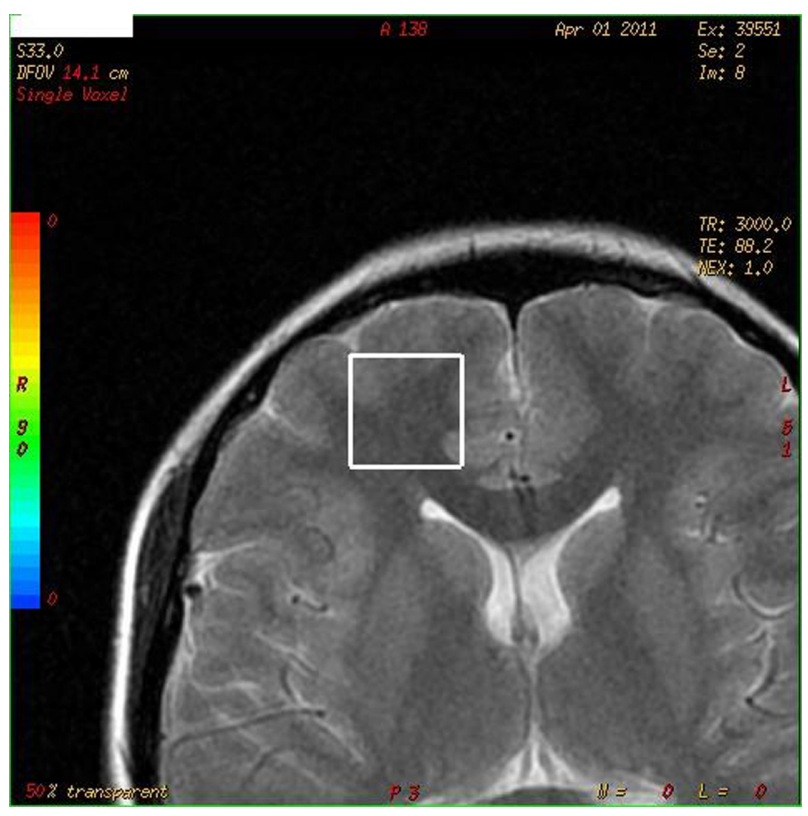

Figure I The right prefrontal region: investigated the neurochemical metabolite levels.

technique was directed at the right PFC region. To ensure that the relevant brain tissue was covered, the volume that was analyzed (volume of interest [VOI]) was manually and visually selected from the relevant regions that were mostly in the frontal lobe. The VOI that the neurochemical metabolites measured was $8 \mathrm{~cm}^{3}$. A chemical shift selective pulse $^{24}$ was used to suppress the signals that originated from water. A point-resolved spectroscopy ${ }^{25}$ technique was then used to localize the volume of the spectroscopy (TR/ TE: $3000 / 144$ and 3000/35 ms). As a result, short- and medium-duration TE spectra were obtained across the VOIs in regions of the right PFC, and these data were evaluated using GE's spectral analysis program. The metabolite ratios were also evaluated.

\section{Statistical analyses}

Statistical analyses of the data were performed using SPSS 15.0 software (IBM SPSS, Armonk, NY, USA). In addition to definitive statistical methods, a Mann-Whitney $U$ test was used to compare the ages, the education levels, and the neurochemical metabolite levels of the depression and control groups. The relationship between HAM-D scores and neurochemical metabolites was analyzed with Pearson correlation analysis. Comparisons of neurometabolite values among the groups (mild MDD, moderate MDD, and healthy control group) were evaluated with nonparametric Kruskal-Wallis tests because the numbers of cases in the groups were not equal and because some values did not follow normal distributions. The significances of the findings 
that were obtained from these tests were examined using Mann-Whitney $U$ tests with Bonferroni corrections. A value of $P<0.016$ was assumed to be significant.

\section{Results}

Thirty-nine patients with MDD and 16 healthy controls participated in the study. The ages and educational levels of the two groups were similar $(P>0.05)$. There were 36 women and 3 men in the group with MDD. There were 12 women and 4 men in the control group. No difference was found between the gender distributions of both groups (chi-square, $P>0.05$ ). HAM-D scores were $15.80 \pm 5.46$ (mean \pm standard deviation [SD]) in all patients with MDD. No differences in the levels of NAA, Cr, Cho, NAA/Cr, and $\mathrm{Cho} / \mathrm{Cr}$ were found between all the patients with MDD and the control group (Table 1).

The ages and the educational levels of the subjects in the mild MDD, moderate MDD, and control groups were similar (Table 2). The HAM-D scores of the moderate MDD group were significantly higher than those of the mild MDD group (respectively, 20.61 $\pm 4.04 ; 12.00 \pm 2.35$; $z=5.207$, $P<0.001$, Mann-Whitney $U$ test). Differences were found in the NAA/Cr levels of the patients with mild MDD, the patients with moderate MDD, and the control group (Kruskal-Wallis test; Table 2). No differences in the levels of NAA, Cr, Cho, and Cho/Cr ratio were found among any of the three groups (Kruskal-Wallis test; Table 2). NAA/Cr levels in the moderate MDD group were lower than those of the control group ( $\mathrm{z}=2.478, P=0.013$; Mann-Whitney $U$ tests with Bonferroni corrections). NAA/Cr levels in the moderate MDD group were lower than those of the mild MDD group, this difference was statistically close to significant levels $(\mathrm{z}=1.930, P=0.055$, Mann-Whitney $U$ tests with Bonferroni corrections). No significant difference between

Table I Comparison of neurochemical metabolites in depression group and control group in prefrontal cortex

\begin{tabular}{lcccc}
\hline Variables & $\begin{array}{l}\text { Depression group } \\
(\mathbf{n}=\mathbf{3 9}) \\
\text { Mean } \pm \text { SD }\end{array}$ & $\begin{array}{l}\text { Control group } \\
(\mathbf{n}=\mathbf{1 6})\end{array}$ & $\mathbf{z}^{*}$ & $\mathbf{P} * *$ \\
& Mean $\pm \mathbf{S D}$ & & \\
\hline Age & $35.82 \pm 12.93$ & $31.31 \pm 5.65$ & 0.909 & 0.363 \\
Education years & $9.51 \pm 4.65$ & $9.43 \pm 4.70$ & 0.363 & 0.985 \\
NAA & $61.71 \pm 17.08$ & $66.37 \pm 9.87$ & 1.548 & 0.122 \\
Cho & $35.68 \pm 7.41$ & $35.31 \pm 6.34$ & 0.052 & 0.958 \\
Cr & $37.98 \pm 9.11$ & $38.75 \pm 6.86$ & 0.306 & 0.759 \\
NAA $/ C r$ & $1.59 \pm 0.33$ & $1.73 \pm 0.21$ & 1.772 & 0.076 \\
Cho/Cr & $0.99 \pm 0.25$ & $0.94 \pm 0.13$ & 0.291 & 0.771
\end{tabular}

Notes: *Mann-Whitney $U$ test; **P $<0.05$ shows significance.

Abbreviations: NAA, $\mathrm{N}$-acetyl aspartate; Cho, choline; $\mathrm{Cr}$, creatine; SD standard deviation; $\mathrm{n}$, number.
NAA/Cr levels in the mild MDD and control groups was found ( $\mathrm{z}=0.924, P=0.356$; Mann-Whitney $U$ tests with Bonferroni corrections).

No correlation was found between neurochemical metabolite levels and HAM-D scores in patients with mild MDD, moderate MDD, and all depression groups (Pearson correlation analysis, for all $P>0.05$ ), or between neurochemical metabolite levels and age (Pearson correlation analysis, for all $P>0.05)$.

\section{Discussion}

In our study, neurochemical metabolite levels in the PFC were investigated in first-episode MDD. The depressive symptom levels of the patients were mild and moderate. The patients were not using any psychotropic medication. No differences in neurochemical metabolite values were found between all patients with MDD and the control group. No correlation was found between neurochemical metabolite levels and HAM-D scores in the patients. NAA/Cr levels in patients with mild MDD were also observed to be similar to the control group. However, we found that the NAA/Cr levels in the patients with moderate MDD were lower than those of the control group. Our results suggest that the neurochemical metabolite level may be abnormal in moderate MDD. Our results lead us to think that depressive symptom intensity can be an important element affecting neuronal functions.

Some studies have reported that levels of neurochemical metabolites such as NAA, Cho, Cr, NAA/Cr, and $\mathrm{Cho} / \mathrm{Cr}$ in the PFC are not different compared to healthy control groups. ${ }^{15,26,27}$ Nery et $\mathrm{al}^{28}$ found that the levels of NAA, Cr, and Cho in the dorsolateral PFC of MDD patients were not different compared to the healthy control group. In the Nery's study, the patients had a mean HAM-D score of $14.8 \pm 6.9$. The distribution of the severity of depression among this patient population was as follows: $30.8 \%$ of patients had mild MDD, $61.5 \%$ had moderate MDD, and 7.7\% had severe MDD. No correlations were found between the severity of the disease, the number of depressive episodes, and neurochemical metabolite levels. These results in our study suggest that neurochemical metabolite levels are not changed in MDD.

In contrast, there have been studies that have reported different results. Wang et $\mathrm{al}^{29}$ reported that levels of NAA/Cr and $\mathrm{Cho} / \mathrm{Cr}$ in the dorsolateral PFC in MDD patients were lower than those of the healthy control group. Patients who had HAM-D scores of 18 and above, who did not use medication, and who had experienced their first depressive episode were evaluated in this study. The severity of depression and the results that were obtained are similar to the characteristics 
Table 2 Comparison of neurochemical metabolites in mild MDD, moderate MDD, and control groups in prefrontal cortex

\begin{tabular}{|c|c|c|c|c|c|}
\hline \multirow[t]{2}{*}{ Variables } & \multirow{2}{*}{$\frac{\text { Mild MDD }(n=2 I)}{\text { Mean } \pm \text { SD }}$} & \multirow{2}{*}{$\frac{\text { Moderate MDD }(n=18)}{\text { Mean } \pm \text { SD }}$} & \multirow{2}{*}{$\frac{\text { Control }(n=16)}{\text { Mean } \pm \text { SD }}$} & \multirow[t]{2}{*}{$\chi^{*}$} & \multirow[t]{2}{*}{$\mathbf{P} * *$} \\
\hline & & & & & \\
\hline Age & $33.80 \pm 12.31$ & $38.16 \pm 13.59$ & $31.31 \pm 5.65$ & $2.06 I$ & 0.357 \\
\hline Education year & $10.90 \pm 3.70$ & $7.88 \pm 5.20$ & $9.43 \pm 4.70$ & 3.573 & 0.168 \\
\hline NAA & $63.67 \pm 18.67$ & $39.56 \pm 11.04$ & $66.40 \pm 10.21$ & 2.448 & 0.294 \\
\hline Cho & $34.43 \pm 8.15$ & $37.26 \pm 7.07$ & $35.31 \pm 6.34$ & $1.89 \mid$ & 0.389 \\
\hline $\mathrm{Cr}$ & $36.63 \pm 7.07$ & $39.56 \pm 11.04$ & $38.75 \pm 6.86$ & 1.386 & 0.500 \\
\hline $\mathrm{NAA} / \mathrm{Cr}$ & $1.67 \pm 0.23$ & $1.49 \pm 0.40$ & $1.75 \pm 0.20$ & 7.552 & 0.023 \\
\hline Cho/Cr & $0.95 \pm 0.24$ & $1.05 \pm 0.25$ & $0.94 \pm 0.13$ & 1.265 & 0.531 \\
\hline
\end{tabular}

Notes: *Kruskal-Wallis test; **P $<0.05$ shows significance. Bold text relates to a statistically significant result.

Abbreviations: NAA, N-acetyl aspartate; Cho, choline; Cr, creatine; DLPFC, dorsolateral prefrontal cortex; HAM-D, Hamilton Rating Scale for Depression; MDD, major depressive disorder; mild MDD, HAM-D score SI5; moderate MDD, HAM-D score > I5; SD, standard deviation; n, number.

and results of our moderate MDD group. In another study, it was determined that the NAA/Cr ratios in the mediofrontal cortex were lower among patients with moderate MDD (the mean HAM-D score was $25.6 \pm 5.0$ ) compared with those in the healthy control group. Furthermore, NAA/Cr levels increased after treatment with antidepressants. This study also found evidence of a relationship between the severity of depression and NAA/Cr levels: the latter decreased as the former increased. There have been studies that have shown that neurochemical changes are affected by the duration of illness, chronicity, and the number of depressive episodes. ${ }^{30}$ In our study, we evaluated first-episode MDD. We determined that NAA/Cr levels were lower in moderate MDD. NAA is a compound recognized as a marker for neuronal integrity, viability, and/or function. $\mathrm{Cr}$ is used for our energy metabolism. Thus, our results indicate decreased neuronal integrity, vitality, and/or nonhealthy neuronal functions or abnormal cellular energy metabolism in moderate-level depression.

Studies in this area have also been done in children and the elderly. The studies in adolescents observed increases in Cho levels in the orbitofrontal cortex in patients with depression. ${ }^{31}$ In 10-16-year-old patients, it was found that Cho levels were higher in the left dorsolateral PFC, but unchanged in the right. It was found that levels of NAA and $\mathrm{Cr}$ were normal in the left and right dorsolateral PFC. ${ }^{32}$ In a study of children with depressive disorder, decreases in NAA levels in the medial PFC and decreases in the levels of NAA and Cho in the anterior cingulate cortex were found. ${ }^{33}$ It was reported that Cho levels were lower, and that levels of NAA and $\mathrm{Cr}$ in the dorsolateral PFC were similar to those of healthy control groups. ${ }^{34}$ In a study of elderly patients with MDD, it was found that patients who did not use antidepressant medication had low NAA/Cr ratios in the frontal white matter compared with the healthy controls. No differences between the patients who used medication and the healthy controls were observed. This study, in which the patients who were evaluated had HAM-D scores that were 15 or higher, also reported that there was no relationship between the severity of depression, the age of disease onset, or neurochemical metabolite levels. ${ }^{35}$ It has been reported that NAA/Cr levels were lower ${ }^{36}$ and $\mathrm{Cho} / \mathrm{Cr}$ levels were higher in patients with late-life depression. ${ }^{37}$ There have been studies showing increases in Cho levels and decreases or no change in NAA and Cr levels in children with MDD; other studies have shown that NAA levels are lower and Cho and $\mathrm{Cr}$ levels are higher in the elderly with MDD. Middle-aged patients with MDD were included in our study. A relationship between age and neurochemical metabolites was not found. Results suggest that neurochemical metabolite levels show similar changes in different age groups in MDD.

Structural and functional abnormalities have been detected in many brain areas in patients with MDD, including areas such as the PFC including the dorsolateral and ventrolateral regions, the hippocampus, the amygdala, and the basal ganglia. It has been suggested that the structural changes that occur in MDD are affected by factors such as age, family history, genetic factors, and the severity of the disease. ${ }^{38}$ Our results support the theory that the severity of depression may be linked to neurochemical metabolite levels. The PFC plays an essential role in mood regulation and cognitive functions, such as executive functions, in MDD. Planning and problem-solving may be impaired in patients with MDD. ${ }^{13,39}$ The relationships between neurochemical changes in the PFC and depressive symptomatology and cognitive function in MDD require further investigation.

Our study has some limitations. One is the lack of cases with severe, recurrent, and chronic MDD. Studies in such patients will contribute to our understanding of the relationship between neurochemical changes and the severity of depression. Another limitation of our study was that the scan was performed with a 1.5 Tesla MRS scanner 
using single-voxel MRS at 3 Tesla and higher is more suitable for detecting levels of neurochemical metabolites. When evaluating such results, the characteristics of the device should be considered for further studies. In addition, absolute concentrations of neurochemical metabolite can be significantly influenced by gray matter and the cerebrospinal fluid content of the VOI. ${ }^{8}$ In addition, the features of depressive episode could be better defined in our study. These were the first depressive episodes of the patients and there was no medication. There were no psychotic findings. However, parameters such as the duration of depressive symptoms could be better defined. These limitations should be considered when evaluating the results of our study.

In conclusion, our results suggest that $\mathrm{NAA} / \mathrm{Cr}$ levels are low in moderate-level MDD. Our results suggest that neuronal function and viability decrease in moderate MDD. There is a need to study the relationship severity of depression and neurochemical metabolites and the effects of severe depression on neuronal functions.

\section{Acknowledgments}

The Scientific and Technological Research Council of Turkey (TÜBİTAK) provided financial support for this study.

\section{Disclosure}

The authors report no other conflicts of interest in this work.

\section{References}

1. Hastings RS, Parsey RV, Oquendo MA, Arango V, Mann JJ. Volumetric analysis of the prefrontal cortex, amygdala, and hippocampus in major depression. Neuropsychopharmacology. 2004;29:952-959.

2. Zou K, Deng W, Li T, et al. Changes of brain morphometry in first-episode, drug-naive, non-late-life adult patients with major depression: an optimized voxel-based morphometry study. Biol Psychiatry. 2010;67:186-188.

3. Malykhin NV, Carter R, Hegadoren KM, Seres P, Coupland NJ. Fronto-limbic volumetric changes in major depressive disorder. J Affect Disord. 2012;136:1104-1113.

4. Rajkowska G, Miguel-Hidalgo JJ, Wei J, et al. Morphometric evidence for neuronal and glial prefrontal cell pathology in major depression. Biol Psychiatry. 1999;45:1085-1098.

5. Narita H, Odawara T, Iseki E, Kosaka K, Hirayasu Y. Psychomotor retardation correlates with frontal hypoperfusion and the Modified Stroop Test in patients under 60-years-old with major depression. Psychiatry Clin Neurosci. 2004;58:389-395.

6. Oh DH, Son H, Hwang S, Kim SH. Neuropathological abnormalities of astrocytes, GABAergic neurons, and pyramidal neurons in the dorsolateral prefrontal cortices of patients with major depressive disorder. Eur Neuropsychopharmacol. 2012;22:330-338.

7. Lorenzetti V, Allen NB, Fornito A, Yücel M. Structural brain abnormalities in major depressive disorder: a selective review of recent MRI studies. J Affect Disord. 2009;117:1-17.

8. Arnone D, McIntosh AM, Ebmeier KP, Munafò MR, Anderson IM. Magnetic resonance imaging studies in unipolar depression: systematic review and meta-regression analyses. Eur Neuropsychopharmacol. 2012;22:1-16.
9. Yıldız-Yeşiloğlu A, Ankerst DP. Neurochemical alterations of the brain in bipolar disorder and their implications for pathophysiology: a systematic review of the in vivo proton magnetic resonance spectroscopy findings. Prog Neuropsychopharmacol Biol Psychiatry. 2006;30:969-995.

10. Monkul ES, Yildız S, Soares JC. Magnetic resonance spectroscopy (MRS) applications in bipolar disorder. Turk Psikiyatri Derg. 2004;15: 138-147.

11. Phillips ML, Ladouceur CD, Drevets WC. A Neural model of voluntary and automatic emotion regulation: implications for understanding the pathophysiology and neurodevelopment of bipolar disorder. Mol Psychiatry. 2008;13:833-857.

12. Michael N, Erfurth A, Ohrmann P, Arolt V, Heindel W, Pfleiderer B. Metabolic changes within the left dorsolateral prefrontal cortex occurring with electroconvulsive therapy in patients with treatment resistant unipolar depression. Psychol Med. 2003;33:1277-1284.

13. Gruber S, Frey R, Mlynarik V, et al. Quantification of metabolic differences in the frontal brain of depressive patients and controls obtained by ${ }^{1} \mathrm{H}-\mathrm{MRS}$ at 3 T. Invest Radiol. 2003;38:403-408.

14. Coupland NJ, Ogilvie CJ, Hegadoren KM, Seres P, Hanstock CC, Allen PS. Decreased prefrontal Myo-inositol in major depressive disorder. Biol Psychiatry. 2005;57:1526-1534.

15. Binesh, N, Kumar A, Hwang S, Mintz J, Thomas, MA. Neurochemistry of late-life depression: a pilot two-dimensional MR spectroscopic study. J Magn Reson Imaging. 2004;20:1039-1045.

16. Grachev ID, Ramachandran TS, Thomas PS, Szeverenyi NM, Fredrickson BE. Association between dorsolateral prefrontal n-acetyl aspartate and depression in chronic back pain: an in vivo proton magnetic resonance spectroscopy study. J Neural Transm. 2003;110:287-312.

17. Milne A, Macqueen GM, Yucel K, Soreni N, Hall GB. Hippocampal metabolic abnormalities at first onset and with recurrent episodes of a major depressive disorder: a proton magnetic resonance spectroscopy study. Neuroimage. 2009;47:36-41.

18. Brambilla P, Stanley JA, Nicoletti MA, et al. 'H-Magnetic resonance spectroscopy study of dorsolateral prefrontal cortex in unipolar mood disorder patients. Psychiatry Res. 2005;138:131-139.

19. Gönül AS, Kitis O, Ozan E, et al. The effect of antidepressant treatment on $\mathrm{N}$-acetyl aspartate levels of medial frontal cortex in drug-free depressed patients. Prog Neuropsychopharmacol Biol Psychiatry. 2006;30:120-125.

20. Horn DI, Yu C, Steiner J, et al. Glutamatergic and resting-state functional connectivity correlates of severity in major depression - the role of pregenual anterior cingulate cortex and anterior insula. Front Syst Neurosci. 2010;4:1-10.

21. American Psychiatric Association. Diagnostic and Statistical Manual of Mental Disorders, Fourth Edition. Washington, DC: American Psychiatric Association; 1994.

22. Hamilton MA. Rating scale for depression. J Neurol Neurosurg Psychiatry. 1960;23:56-62.

23. Akdemir A, Türkçapar MH, Örsel SD, Demirergi N, Dag I, Ozbay MH. Reliability and validity of the Turkish version of the Hamilton Depression Rating Scale. Compr Psychiatry. 2001;42:161-165.

24. Kienlin von M.The basics of magnetic resonance spectroscopy. In:Methodology, spectroscopy and clinical MRI.15th Annual Scientific Meeting 1998;3-7.

25. Klose U. Measurement sequences for single voxel proton MR spectroscopy. Eur J Radiol. 2008;67:194-201.

26. Kaymak SU, Demir B, Oğuz KK, Sentürk S, Uluğ B. Antidepressant effect detected on proton magnetic resonance spectroscopy in drugnaïve female patients with first-episode major depression. Psychiatry Clin Neurosci. 2009;63:350-356.

27. Auer DP, Pütz B, Kraft E, Lipinski B, Schill J, Holsboer F. Reduced glutamate in the anterior cingulate cortex in depression: an in vivo proton magnetic resonance spectroscopy study. Biol Psychiatry. 2000;47:305-313.

28. Nery FG, Stanley JA, Chen HH, et al. Normal metabolite levels in the left dorsolateral prefrontal cortex of unmedicated major depressive disorder patients: a single voxel (1) H spectroscopy study. Psychiatry Res. 2009;30:177-183. 
29. Wang Y, Jia Y, Xu G, Ling X, Liu S, Huang L. Frontal white matter biochemical abnormalities in first-episode, treatment-naive patients with major depressive disorder: A proton magnetic resonance spectroscopy study. J Affect Disord. 2012;136:620-626.

30. Portella MJ, de Diego-Adeliño J, Gómez-Ansón B, et al. Ventromedial prefrontal spectroscopic abnormalities over the course of depression: a comparison among first episode, remitted recurrent and chronic patients. J Psychiatr Res. 2011;45:427-434.

31. Steingard RJ, Yurgelun-Todd DA, Hennen J, et al. Increased orbitofrontal cortex levels of choline in depressed adolescents as detected by in vivo proton magnetic resonance spectroscopy. Biol Psychiatry. 2000;48:1053-1061.

32. Farchione TR, Moore GJ, Rosenberg DR. Proton magnetic resonance spectroscopic imaging in pediatric major depression. Biol Psychiatry. 2002;52:86-92.

33. Olvera RL, Caetano SC, Stanley JA, et al. Reduced medial prefrontal $\mathrm{N}$-acetyl-aspartate levels in pediatric major depressive disorder: a multivoxel in vivo (1)H spectroscopy study. Psychiatry Res. 2010;184: 71-76.

34. Caetano SC, Fonseca M, Olvera RL, et al. Proton spectroscopy study of left dorsolateral prefrontal cortex in pediatric depressed patients. Neurosci Lett. 2005;384:321-326.
35. Henigsberg N, Bajs M, Hrabac P, et al. Changes in brain metabolites measured with magnetic resonance spectroscopy in antidepressant responders with comorbid major depression and posttraumatic stress disorder. Coll Antropol. 2011;35(Suppl 1):145-148.

36. Chen CS, Chiang IC, Li CW, et al. Proton magnetic resonance spectroscopy of late-life major depressive disorder. Psychiatry Res. 2009; 172:210-214.

37. Kumar A, Thomas A, Lavretsky H, et al. Frontal white matter biochemical abnormalities in late-life major depression detected with proton magnetic resonance spectroscopy. Am J Psychiatry. 2002;159:630-636.

38. Holtzheimer PE, Mayberg HS. Neuropsychiatric aspects of mood disorders. In: Yudofsky SC, Hales RE, editors. The American Psychiatric Publishing Textbook of Neuropsychiatry and behavioral Neurosciences. Fifth Edition, Washington DC. 2008.

39. Fossati P, Ergis AM, Allilaire JF. Executive functioning in unipolar depression: a review. Encephale. 2002;28:97-107.
Neuropsychiatric Disease and Treatment

\section{Publish your work in this journal}

Neuropsychiatric Disease and Treatment is an international, peerreviewed journal of clinical therapeutics and pharmacology focusing on concise rapid reporting of clinical or pre-clinical studies on a range of neuropsychiatric and neurological disorders. This journal is indexed on PubMed Central, the 'PsycINFO' database and CAS.

\section{Dovepress}

The manuscript management system is completely online and includes a very quick and fair peer-review system, which is all easy to use. Visit http://www.dovepress.com/testimonials.php to read real quotes from published authors. 